\title{
The space environment before the space age
}

Article

Published Version

Barnard, L., Owens, M. and Scott, C. (2017) The space environment before the space age. Astronomy \& Geophysics, 58 (2). 2.12-2.16. ISSN 1366-8781 doi:

https://doi.org/10.1093/astrogeo/atx056 Available at https://centaur.reading.ac.uk/72501/

It is advisable to refer to the publisher's version if you intend to cite from the work. See Guidance on citing.

Published version at: http://dx.doi.org/10.1093/astrogeo/atx056

To link to this article DOI: http://dx.doi.org/10.1093/astrogeo/atx056

Publisher: Oxford University Press

All outputs in CentAUR are protected by Intellectual Property Rights law, including copyright law. Copyright and IPR is retained by the creators or other copyright holders. Terms and conditions for use of this material are defined in the End User Agreement.

\section{www.reading.ac.uk/centaur}

\section{CentAUR}

Central Archive at the University of Reading

Reading's research outputs online 


\section{The space environment before the space age}

\author{
Luke Barnard, Mat Owens and \\ Chris Scott sum up a meeting \\ that focused on ways to stretch \\ the space environment record \\ back into the past.
}

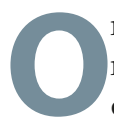
n 10 February 2017, the Royal Astronomical Society hosted a specialist discussion meeting on "Understanding the space environment before the space age". This meeting reviewed recent developments in our understanding of space weather and space-climate variability derived primarily from historical observations, or from proxy records of past solar activity. Most of our knowledge about the heliospheric environment, particularly space weather and space climate, is based on the analysis of observations recorded by spacecraft and terrestrial instruments operating in the space age. These modern data have driven rapid progress in solar and heliospheric physics, and will continue to be the mainstay of these research fields into the future. However, solar and geophysical observations have been recorded for centuries, often diligently and routinely, and the fingerprints of past solar activity remain embedded in the geophysical record through, for example, concentrations of cosmogenic radionuclides in ice sheets and tree trunks.

There is significant value in these historical observations and proxy records: they provide unique insights into space-weather events and space-climate variability not observed over the modern epoch of comprehensive solar and heliospheric observations. For example, it is well documented that extreme space-weather events still took place before the space age and at more moderate levels of solar activity. The Carrington event of September 1859 (Hodgson 1859 , Carrington 1859) is probably the most famous example of such an extreme spaceweather episode. Analysis of the available solar and geomagnetic data reveal that this sequence of activity consisted of what was probably the most intense directly observed solar flare (rated X45 \pm 5 ), followed by an extreme geomagnetic storm and remarkable aurora, including sightings from equatorial regions (Cliver \& Dietrich 2013). The Carrington event is frequently used as a worst-case scenario in spaceweather mitigation studies (Hapgood 2011), and this is only possible because of the recording and analysis of these historical geophysical observations.

Furthermore, several independent estimates of the Sun's magnetic activity demonstrate that the space age has coincided with a period of unusually high solar activity, often termed a grand solar maximum (Owens et al. 2016ab, Usoskin et al. 2016). Based on the analysis of cosmogenic radionuclides reconstructions of past solar activity, predictions have been made ....... that the current grand solar "Many historic data are maximum is ending (Abreu et al. 2008, Barnard et al. 2011, Steinhilber \& Beer 2013), with observations of solar cycle 24 being, so far, consistent with this (Lockwood et al. 2012). Therefore, it is reasonable to question how representative the knowledge gleaned from these spaceage observations will be upon a return to more moderate levels of solar activity.

\section{Variety of sources}

There are many sources of potentially under-exploited observations, including terrestrial magnetometers, neutron monitors, ionization chambers, ionosonde retrievals, cosmogenic iosotope depositions, and atmospheric electricity measurements. These quantitative data are supplemented by sunspot drawings, eclipse observations of the solar corona, records of auroral sightings and reports of the impacts of space-weather events. However, as detailed below, some of these records are difficult to find, tricky to interpret, and may be stored on vulnerable or cumbersome media such as paper records and photographic plates. A key challenge lies in sourcing and preserving these data before they are lost or irreparably damaged. Here we provide an overview of the presentations made by the speakers at the discussion meeting, as well as points of discussion that arose throughout the day.

Mike Hapgood (RAL Space) began the morning by discussing space-weather risk, particularly relating to the UK, and how mitigation requires relevant extreme event scenarios. Societies are becoming 


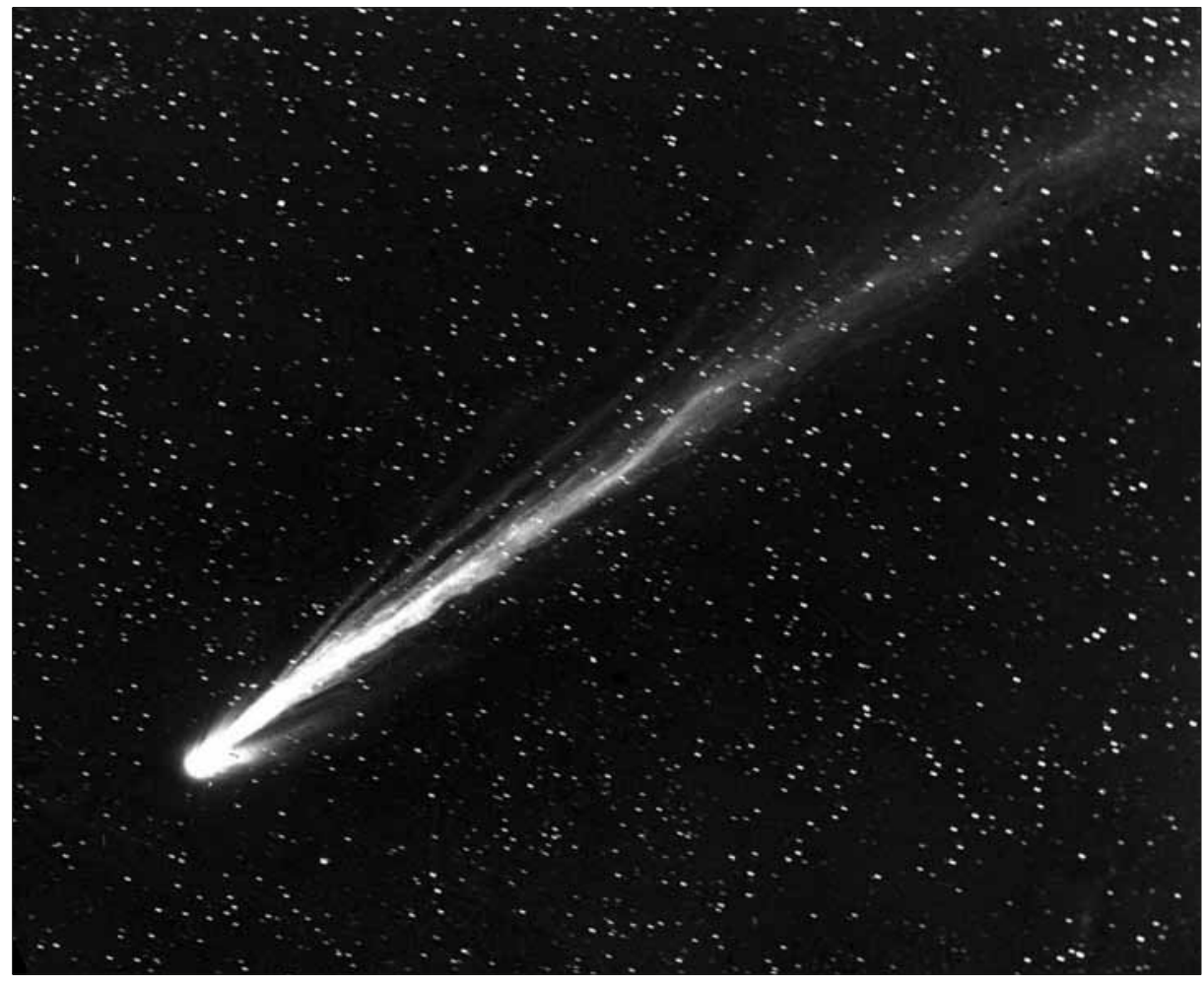

1 Photograph of Comet Morehouse, C/1908 R1. The comet's plasma tail shows signs of detailed structure due to interaction with the solar-wind plasma. At the meeting, Geraint Jones discussed how this plasma tail structure may be used to infer properties of the solar wind.

increasingly sensitive to space-weather phenomena, driven by a general increasing reliance on technological systems (Hapgood 2011). As an example, consider the development of railway systems. At the turn of the 20th-century, railways relied on steam locomotives and manually operated railway switches, both of which were robust against space weather. Now, increasingly, trains are electrically powered, tracks are electrically controlled, and navigation and routing may depend on global navigation satellite systems (GNSS). Each of these factors are sensitive to a range of space-weather phenomena: ground-induced currents (GICs) are a risk to electrical power distribution, while GNSS rely on electronics that can be disrupted by solar energetic particles, cosmic rays and radiation belt particles, as well as effective communication of the GNSS signals, which can be affected by dynamic changes in ionospheric structure and composition during geomagnetic storms.

Currently, the main focus is on understanding and mitigating against the effects of severe events that pose risks to critical infrastructures. This is often achieved through the analysis of extreme event scenarios developed by considering the statistics of past events and analysing the impacts of, for example, a 1-in-100 or 1-in1000-year event, analogous to our approach to other natural hazards such as flooding. However, Hapgood emphasized that, in addition to this empirical approach, it is also vital to pursue a better understanding of the underlying physics, which may allow us to assess how plausible empirically derived extreme event scenarios are, particularly if an event magnitude is extrapolated from a statistical model beyond the observed range. Furthermore, observations of Sun-like stars may provide another opportunity to improve our understanding of the physics of space-weather phenomena. Finally, he highlighted a concern that

...... many historic data are at risk "There are plans to of being lost due to being use the new software stored in analogue forms. He to reanalyse archival also stressed that while these data remain in this state they are of limited use. The talk concluded with a suggestion that our understanding of extreme space weather could be improved if these historic data were protected by consolidating them into modern, usable formats, and made discoverable for use throughout the community.

Geraint Jones (Mullard Space Science Laboratory, University College London) described how comets can be used as tracers of solar-wind activity before the space age. Comets propagating through the inner solar system produce both a dust tail and an ion tail. The ion tail is strongly affected by interaction with the solar-wind plasma flow and the entrained interplanetary magnetic field. In fact, early photographs of cometary tails provided the first evidence of the existence of a solar wind; the ion tail of a comet flows approximately radially away from the Sun with the solar wind, deflected away from the radial by a small angle because of the comet's orbital motion (Biermann 1951). The first known photograph of a comet was taken in 1858, of Comet Donati, and the skill of comet photography quickly increased. One such instance is that of Comet Morehouse, C/1908 R1, photographed in 1908 (figure 1). The ion tail extends diagonally across the majority of the image frame, and appears to show detailed structure resulting from the interaction with the solar-wind flow.

Analysis of the direction of the ion tail relative to the anti-sunward radial and the comet's orbital motion can provide estimates of the solar-wind speed local to the comet. Furthermore, tail-disconnection events, when the comet's ion tail appears to separate from the coma, are thought to occur when the comet propagates through the heliospheric current sheet (HCS), and perhaps when interacting with coronal mass ejections. Therefore, records of taildisconnection events can provide estimates of the location of the HCS and solar-wind transients. Estimates of the HCS location, particularly its maximum latitudinal reach, are potentially valuable as they can provide a measure of the magnetic complexity of the corona and the progression of the solar cycle polarity reversal. For example, the inclination of the HCS relative to the solar equator is known to be a useful predictor of the phase of the 11-year Schwabe solar cycle (Owens et al. 2011), which is difficult to determine from sunspot records alone.

Jones described an archive of solarwind speed estimates from the analysis of 1600 observations of comet tails (Belton \& Brandt 1966). Recent work by Ramanjooloo $(2014,2015)$ has produced software to analyse comet tail observations, which will be released to the wider community in the near future. Furthermore, there are plans to use the new software to reanalyse archival images of comet tails. Discussion focused on whether temporal and spatial variability in observations of comet tails could be used to infer the level of turbulence in the solar wind, as well as structure in the solar-wind flow.

\section{Boat as ice station}

Julia Wilkinson (University of Reading) summarized the results of her investigation into the auroral observations from the ill-fated expedition of the USS Jeannette, which became trapped in Arctic ice for two years between 1879 and 1881 (Wilkinson et al. 2016). The Jeannette was a United States Navy expedition aiming to sail to the North Pole, to claim it as a United States territory. However, a further goal of the mission was to observe the aurora and measure associated electrical currents 
induced at ground level. Trapped for two years in freezing and perilous conditions, the crew embraced its role aboard what was effectively the first ice station and diligently carried out auroral observations. Citizen-science volunteers taking part in the Old Weather project (http://www.oldweather.org, a Zooniverse project) recently digitized the logbooks of the Jeannette, which contained many of these auroral observations. By examining the ship's logs, together with additional notes from the captain's journal and the executive officer's notebook, a surprisingly detailed picture of Arctic auroral activity emerges.

Wilkinson presented a chart that summarizes the Jeannette's auroral observations (figure 2). The beginning and end dates of two auroral seasons can be easily identified. But more subtly, it is also possible to discern the effect of the lunar phase on the visibility of displays; auroral displays are clearly reduced around the dates of full Moon. The times that the auroral displays were observed are consistent with our understanding of auroral substorms and it was possible to corroborate the position of the auroral oval by looking at the direction in which the aurora was observed together with information on geomagnetic storms and the geomagnetic aa-index. There was also some evidence for recurrent auroral activity, in that repeated colourful auroral observations coincided with records of large sunspot groups recorded at the Royal Observatory Greenwich.

The Jeanette eventually succumbed to the ice and, tragically, only 13 of the crew of 33 survived. At a time when little was known about the cause of auroral activity, the Jeannette gathered valuable information that was never published as had been intended. Now, 136 years later, these records provide a detailed insight into Arctic aurorae in the early 1880s, demonstrating that hidden historical data from an unexpected source can still inform our understanding of space weather.

Electrical currents associated with the auroral observations were recorded by the crew of the Jeanette with a galvanometer, which may constitute some of the earliest measurements of ground-induced currents. However, at present insufficient information has been obtained about the galvanometer to calibrate the observations. It is possible that this metadata is stored elsewhere, and may yet be recovered. However, this perhaps serves as a reminder of the importance of modern data standards: for data to be useful for future generations, it is not only important to store data in a secure way, but also to store with it all necessary metadata to allow proper interpretation. Wilkinson's presentation prompted a discussion about whether

\begin{tabular}{|c|c|c|c|c|c|c|c|c|c|c|c|c|c|c|c|c|c|c|c|c|c|c|c|c|c|c|c|c|c|}
\hline 1 & 1 Sep & F & & & & & & & & & & & & & & & & & & & & & & & & & & & 27 Sep \\
\hline 8 & 28 Sep & & & F & & & & & & & & & & & & & & & & & & & & & & & & & $240 \mathrm{ct}$ \\
\hline 7 & $250 \mathrm{oct}$ & & & & & F & & & & & & & & & & & & & & & & & & & & & & & $20 \mathrm{Nov}$ \\
\hline \multirow[t]{5}{*}{9} & 21 Nov & & & & & & & F & & & & & & & & & & & & & & & & & & & & & $17 \mathrm{Dec}$ \\
\hline & $18 \mathrm{Der}$ & & & & & 2 & 2. & & & & F & & & & & & Z & & & & & Z & & & & $z$ & & Z & 13 latt \\
\hline & $14 \mathrm{Jn}$ & & 2 & F & $z$ & Z & & & & & & & F & & & & $\mathbf{Z}$ & Z & & & & & & 2 & $Z$ & & 22 & Z & 9 Feb \\
\hline & $10 \mathrm{Feb}$ & $z$ & 2 & & & & & $z$ & $z$ & & & $\mathbf{Z}$ & & & & 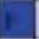 & & & & & $\mathbf{z}$ & Z & & $z$ & $Z$ & 2 & $z$ & & 7 Mar \\
\hline & 8 Mar & $z$ & $\mathbf{z}$ & & $z$ & 2 & & $z$ & & Z & $z$ & & & $\mathbf{Z}$ & & & & F & Z & & & & & & & & & & 3 Apr \\
\hline 1 & $4 A_{p K}$ & & $\mathbf{z}$ & & & & & & & & & & & & & & & & & & F & & & & & & & & $30 \mathrm{Apr}$ \\
\hline 8 & I May & & & & & & & & & & & & & & & & & & & & & & & F & & & & & 27 May \\
\hline 8 & $28 \mathrm{May}$ & & & & & & & & & & & & & & & & & & & & & & & & & F & & & 23 Jun \\
\hline \multirow[t]{11}{*}{0} & $24 \mathrm{Jun}$ & & & & & & & & & & & & & & & & & & & & & & & & & & & & $20 \mathrm{sd}$ \\
\hline & $21 \mathrm{Jul}$ & F & & & & & & & & & & & & & & & & & & & & & & & & & & & 16 Avg \\
\hline & 17 AES & & & & F & & & & & & & & & & & $z$ & & & & & & & & & & & & Z & $12 \mathrm{sep}$ \\
\hline & 13 sep & & & $\mathrm{z}$ & & & F & & & & & & & & Z & & & & & & & & & & 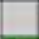 & & & & $90 \mathrm{cr}$ \\
\hline & $100 \mathrm{ct}$ & & & & & & & & $z$ & F & & & & & $z$ & & & & & & & & & & $z$ & & & & $5 \mathrm{Nov}$ \\
\hline & $6 \mathrm{Nov}$ & & $\mathbf{z}$ & & $z$ & & & $z$ & & & & F & & & & & & & $z$ & & & & & & Z & $z=$ & $\mathrm{Z}$ & z & $2 \mathrm{Dec}$ \\
\hline & $3 \mathrm{Dec}$ & $\mathbf{z}$ & & 2. & 2 & & $z$ & & $z$ & $z$ & $z$ & & & & F & & Z & $z$ & & & & & $\mathbf{z}$ & $\mathbf{Z}$ & $z$ & 2 & 2 & & $29 \mathrm{Dec}$ \\
\hline & $30 \mathrm{Dec}$ & & $z$ & & $z$ & Z & & $z$ & & & & $\mathrm{Z}$ & & & & & F & & & & & Z & & 7 & $z$ & 2 & & & 25 Jan \\
\hline & $26 \mathrm{Jm}$ & & & & & & & $z$ & & & $z$ & $\mathrm{z}$ & & Z & & & & & F & & & 7 & 7 & $z$ & & $\mathrm{Z}$ & 72 & Z & $21 \mathrm{Feb}$ \\
\hline & $22 \mathrm{Feb}$ & $z$ & $\mathrm{z}$ & Z & $z$ & & $z$ & & $z$ & $z$ & & $z$ & 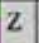 & $\mathrm{z}$ & & & & & $z$ & $z$ & & F & $z$ & & & & & & $20 \mathrm{Mar}$ \\
\hline & 21 Mar & & 2 & & $z$ & Z & & Z & & & $\mathbf{Z}$ & & & & & & & & & & & & & F & & & & & $16 \mathrm{Apr}_{\mathrm{p}}$ \\
\hline 1 & 17 Apr & & & & & & & & & & & & & & & & & & & & & & & & & & F & & 13 May \\
\hline 8 & $14 \mathrm{May}$ & & & & & & & & & & & & & & & & & & & & & & & & & & & & 9 Jin \\
\hline 8 & $10 \mathrm{Jun}$ & & $\mathbf{F}$ & & & & & & & & & & & & & & & & & & & & & & & & & & $6 \mathrm{Jd}$ \\
\hline \multirow[t]{3}{*}{1} & 73 ul & & & $\mathrm{F}$ & & & & & & & & & & & & & & & & & & & & & & & & & 2 Aag \\
\hline & 3 Aug & & & & & & F & & & & & & & & & & & & & & & & & & & & & & $29 \mathrm{~A}=3$ \\
\hline & 30 Ang & & & & & & & & F & & & & & & & & & & & & & & & & & & & & $26 \mathrm{Sep}$ \\
\hline
\end{tabular}

2 A charted summary of the auroral activity observed by the USS Jeannette crew. Each cell represents one day, each row consists of 27 cells, and so shows the auroral activity over a Bartels rotation. Red, yellow, green, purple and white represent colours observed; blue indicates multiple colours; $F=$ full Moon; Z=zenith. (Reproduced from Wilkinson et al. 2016)

other expeditions may also contain useful geophysical observations. The answer is almost certainly yes, and the community can help obtain them by participating in projects like Old Weather.

\section{Solar activity from cosmogenic isotopes}

Ilya Usoskin (University of Oulu, Finland) reviewed the solar activity reconstructed from the cosmogenic isotopes ${ }^{14} \mathrm{C}$ and ${ }^{10} \mathrm{Be}$ in order to determine the upper-limits of the magnitude of past solar energetic

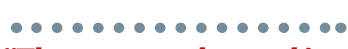
"The crew embraced its role aboard what was effectively the first ice station" particle events. Sequences of solar flares and coronal mass ejections can produce populations of energetic charged particles, known as solar energetic particles (SEPs). Fluxes of SEPs are a significant space-weather hazard, and the most energetic events cause increases in the flux of energetic particles observed by terrestrial instruments, known as ground level enhancements (GLEs). The cosmogenic isotopes ${ }^{14} \mathrm{C}$ and ${ }^{10} \mathrm{Be}$ are produced in the upper atmosphere by nuclear reactions of atmospheric constituents with energetic particles, primarily galactic cosmic rays, which are then stored in tree trunks and ice sheets, respectively. Typically, SEP fluxes are both too low and consist of particles of insufficient energy to produce significant quantities of cosmogenic isotopes. However, modelling of ${ }^{14} \mathrm{C}$ and ${ }^{10} \mathrm{Be}$ production suggests that the largest GLEs can be a significant source of these isotopes, relative to the production by galactic cosmic rays. Analysis of an annual record of ${ }^{14} \mathrm{C}$ concentrations reveals impulsive enhancements in AD775 and AD994 (Miyake et al. 2012, 2013), which have been assessed to be the result of extra ${ }^{14} \mathrm{C}$ production from SEP fluxes (Usoskin et al. 2013).

Inverse modelling of the cosmogenic isotope production for these events provided an estimate of the total SEP flux required to produce these enhancements. The total SEP flux was significantly higher than events observed during the space age, and it was argued that the AD775 and AD994 events may therefore make better worst-case scenarios than the largest SEP events in recent decades. By analysing a ${ }^{14} \mathrm{C}$ record that extends back 10000 years, Usoskin argued that there are approximately 20 candidate events similar to the AD775 event that warrant further investigation. Furthermore, the apparent lack of impulsive enhancements much larger than the AD775 event was used to estimate an upper limit on the 


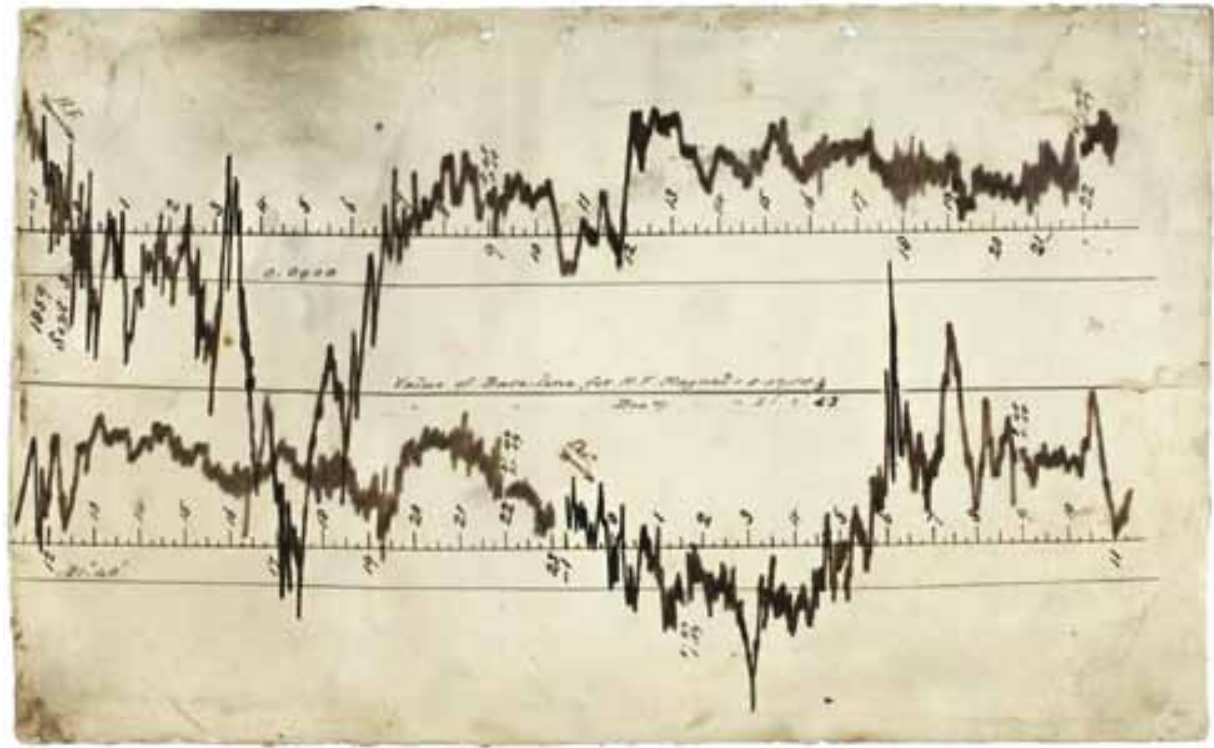

3 This digitized magnetograph, available online via the BGS archive, is from Greenwich observatory, 3 September 1859, near the end of the geomagnetic storm associated with the Carrington event. The top and bottom traces correspond to the horizontal component and declination of the magnetic force, respectively.

maximum SEP event magnitude likely to be seen on timescales of 10000 years.

Until recently, it had also been thought that nitrate concentrations in polar ice cores could serve as a proxy of past SEP events. However, updated analysis of the nitrate records and modelling of nitrate production by SEP fluxes has largely invalidated this proxy record (Wolff et al. 2012, Duderstadt et al. 2014). Usoskin presented additional evidence from the cosmogenic record that nitrate concentrations in polar ice cores are not a ...................... The aa-index is in fact variability in sea-level pressure" valid proxy for SEP fluxes. By assuming that the nitrate proxy record was indeed valid, it is possible to model what the coincident response of the cosmogenic record would have been. This modelling suggests that a large response should have been observed in annually integrated ${ }^{10} \mathrm{Be}$ production, which is not present in any of the available records.

\section{Dating uncertain chronologies}

Ben Pope (University of Oxford) continued the discussion of the cosmogenic isotope records from a different perspective, by considering how impulsive enhancements in the ${ }^{14} \mathrm{C}$ record may be used for archaeological studies, establishing well defined "tie-points" for dating previously uncertain chronologies (Dee \& Pope 2016). Determining absolute dates in history becomes uncertain beyond approximately $800 \mathrm{BC}$. Although the relative timing within periods before this can often be established, these chronologies are said to be "floating" - they cannot be tied to the BC-AD timeline. Pope described a strategy outlined in Dee \& Pope (2016) to resolve these ambiguities through impulsive enhancements in the ${ }^{14} \mathrm{C}$ record, such as the AD755 and AD994 events. By measuring the ${ }^{14} \mathrm{C}$ concentrations in the annual tree rings of wood samples (e.g. in wooden beams of buildings), it is possible to match the date of the impulsive ${ }^{14} \mathrm{C}$ enhancement to a specific tree ring. This method essentially takes advantage of detailed structure within the ${ }^{14} \mathrm{C}$ record to improve the accuracy of standard ${ }^{14} \mathrm{C}$ dating procedures, and has already been successfully employed for dating features around the AD775 event.

However, for this strategy to be of wider use, it is necessary to establish a larger set of impulsive enhancements, particularly for times before the AD775 event. Gaussian process modelling of the decadal ${ }^{14} \mathrm{C}$ record was used to search for candidate events. Pope highlighted that the technical difficulty and expense of measuring ${ }^{14} \mathrm{C}$ on this scale means it is often impractical to perform this for annual tree rings over extended time periods. However, the results of the Gaussian process modelling on the decadal series suggest that candidate events could be identified in lower resolution data; the annual tree rings could then be subsequently measured in detail, potentially making the process significantly cheaper.

Indrani Roy (University of Exeter) described the results of her recent study that compares the influence of sunspot activity and geomagnetic activity on winter surface climate (Roy et al. 2016). Multiple linear regression was used to assess the relative contributions of solar variability, volcanic activity and well-established modes of climate variability (for example, El Nino-Southern Oscillation), on winter averages (from December to February) of pressures at sea level across the globe, over the period 1900-2012. Solar activity was assessed using both the sunspot number and the geomagnetic aa-index. The aaindex is computed from observations of the variability in the geomagnetic field at two approximately antipodal geomagnetic observatories, and is designed to be sensitive to the response of Earth's magnetosphere to variations in the solar wind. Roy pointed out that previous studies have used a similar approach, but assessed solar variability only through the sunspot number.

It was argued that, over the studied period, the aa-index is in fact a stronger predictor of variability in sea-level pressure, particularly in localized areas of the globe. The mechanism by which solar variability affects sea-level pressure in this manner was not discussed. Subsequent discussion focused on the challenges of resolving the effects of solar variability on Earth climate using statistical techniques alone.

Ellen Clarke (British Geological Survey) provided a report on the role of historical British geomagnetic measurements in quantifying space weather and its impact. The British Geological Survey (BGS) maintains an archive of these historical geomagnetic measurements, which have been continuously recorded from various observatories for 175 years, beginning at Greenwich in 1841. The original system for recording continuous magnetometer observations used a suspended magnetmirror arrangement to reflect light onto a rotating reel of photographic paper (e.g. Brooke 1843a,b). These were supplemented by regular absolute measurements, and the photographic traces were sometimes also interpolated into tabulated series.

It is estimated that there are approximately 290000 magnetographs in the archive. The condition of the archive was assessed in 2004 and was found to be potentially at risk, so the BGS began a programme to preserve and digitize the archive of both magnetographs and the observatory yearbooks. This extensive work was completed in 2014, with the remaining records now securely stored. The digitized records are publicly available in an online archive (http://www.bgs. ac.uk/data/Magnetograms/home.html).

An example of a magnetograph image is shown in figure 3 , showing a geomagnetic storm associated with the Carrington event. At present, researchers typically have access only to hourly averages or hourly spot values of geomagnetic data before the introduction of modern digital recording. Figure 3 shows there is much variability in the geomagnetic field on subhourly timescales. Clarke discussed how the BGS is also researching methods to process the magnetograph images to extract high-resolution time series of the magnetic 
observations (for example, 1 minute). Some space-weather effects, such as GICs, depend on variations in the geomagnetic field on timescales shorter than an hour, so these higher resolution estimates may enable investigation of the potential impacts of historic geomagnetic storms.

Clarke also pointed out that these geomagnetic observations are useful for more than space-weather science; they also contribute to our understanding of long-term trends in solar activity and the geodynamo that sustains Earth's internal magnetic field. For example, geomagnetic observations exhibit a diurnal variation arising from current systems in the ionosphere that depend heavily on solar EUV irradiation. The amplitude of the diurnal variation in geomagnetic activity depends on the solar EUV flux, and consequently long-term trends in the amplitude of the diurnal cycle can provide information on solar EUV variability before reliable instrumental records are available.

\section{Naked-eye observations}

David Willis (University of Warwick) presented an interesting analysis of historical naked-eye observations of sunspots and aurorae over the past two-and-a-half millennia. He considered sources from societies spanning the globe, including England, Korea, China, Russia and Babylon, and demonstrated that, by considering these data in unison, a picture emerges of past solar activity that is consistent with our modern understanding of space-weather episodes. One such episode occurred in AD1128, involving perhaps the earliest recorded sunspot drawing. On 8 December AD1128, two large sunspots were observed from England and recorded in a drawing in The Chronicle of John of Worcester (Stephenson \& Willis 1999). Both spots were drawn near the central meridian, with a larger spot in the northern hemisphere and a smaller spot in the southern hemisphere. Five days later, on 13 December, Korean records report that "From the NW to the SW, a red vapour soared and filled the sky". Linking such different records highlights how, because of the difficulty of sourcing, interpreting and often translating these historical texts, such analyses are necessarily collaborative and cross-disciplinary. Willis was careful to point out that there are challenges in interpreting these historical records, such as perhaps not knowing the meteorological conditions, which may complicate, aid, or obscure sunspot and aurorae observations. For example, observations of sunspots from Russia in the years AD 1365 and 1371 were thought to have been aided by nearby forest fires, the smoke from which would dim the Sun, enabling dark spots to be seen with the unaided eye. The recent results of Hayakawa et al. (2017) were discussed, including what are thought to be the earliest known drawings of datable aurorae and cometary tails from the late 8th century, obtained from the analysis of The Chronicle of Züqnin. Considering the availability of historical sources, it was concluded that after approximately AD 1000 records of sunspot and aurorae are sufficiently dense that it becomes possible to build a robust picture of space-weather episodes, which may provide important context for information gleaned from other measures - such as the cosmogenic isotope records. Willis concluded, from his analysis of the available sources, that there is evidence of intense solar activity around AD1128, 1365, 1371 and 1770.

\section{Sunspots and geomagnetic activity}

Mike Lockwood (University of Reading) concluded the meeting with a discussion of reconstructing space-climate conditions for the past 400 years, using results recently published by Owens et al. (2017). He reviewed how heliospheric parameters such as the solar-wind speed and interplanetary magnetic field (IMF) can be reconstructed at annual resolution from a combination of sunspot and geomagnetic activity records. Lockwood described why variations in the efficacy of solarwind-magnetosphere coupling mean that sub-annual resolution reconstructions are unlikely to be obtained. The unknown ratio between the geo-effective component of the IMF and the IMF magnitude averages to constant value only on annual timescales, for which it causes an uncertainty of less than 10\% (Lockwood et al. 2016).
Annual averages of the open solar magnetic flux, near-Earth IMF magnitude, as well as latitudinal profiles of the solar-wind speed and density were derived from a continuity model of the Sun's open magnetic flux. The model requires the sunspot number as an input, but has been calibrated by comparison with approximately 40 years of magnetohydrodynamic modelling of the solar corona using magnetographs. These results suggest that during the Maunder minimum the width of the streamer belt increased and on average the solar wind at Earth was slower and denser, ........ while the IMF magnitude while the suppressed.

The results of Owens et al. (2017) were extended by using solar-wind-magnetosphere coupling functions liospheric modelling results to also provide estimates of the Ap geomagnetic index. The annual mean Ap index was predicted to be suppressed by a factor of approximately two during the Maunder minimum, relative to the modern epoch. Furthermore, since observations began in 1932, the shape of the occurrence distribution of Ap has remained almost constant, despite changing mean values, indicating that there is a strong relationship between the means and the fraction of time when Ap exceeds a large threshold. This was used in conjunction with the heliospheric modelling to estimate the annual fraction of days with large geomagnetic storms, revealing long periods through the Maunder minimum with essentially no large storms predicted.

The meeting was drawn to a close with a short discussion, in which Clare Foullon (University of Exeter) made an announcement about perhaps the earliest recorded space-weather impact. On 18 October 1841, there are reports that a train was delayed travelling between Exeter and Starcross "on the account of a very intense magnetic disturbance". Clare wishes to investigate this event in more detail, and has requested that anyone with more information on this event should please contact her. $\bullet$

\section{AUTHORS}

Luke Barnard, Mat Owens and Chris Scott are based in the space and atmospheric electricity group in the Department of Meteorology at the University of Reading, UK.

\section{REFERENCES}

Abreu JA et al. 2008 Geophys. Res. Lett. 35(20) 2

Barnard LM et al. 2011 Geophys. Res. Lett. 38(16) Belton MJS \& Brandt JC 1966 Astrophys. J. Suppl. Ser. 13125

Biermann L 1951 Z. Astrophys. 29274

Brooke C 1843a Proc. R. Soc. Lond. 13759
Brooke C 1843b Proc. R. Soc. Lond. 13769 Carrington RC 1859 Mon. Not. R. Astron. Soc 20(1) 13

Cliver EW \& Dietrich WF 2013 J. Sp. Weather Sp.

Clim. 3 A31

Dee MW \& Pope BJS 2016 Proc. R. Soc. A Math

Phys. Eng. Sci. 472(2192) 20160263

Duderstadt KA et al. 2014 J. Geophys. Res. Atmos. 119(11) 6938

Hapgood MA 2011 Adv. Sp. Res. 47(12) 2059

Hayakawa H et al. 2017 Sol. Phys. 292(1) 12

Hodgson R 1859 Mon. Not. R. Astron. Soc. 20(1) 15

Lockwood M et al. 2012 Astron. \& Geophys. 533.09

\author{
Lockwood M et al. 2016 Sp. Weather 14(6) 406 \\ Miyake F et al. 2012 Nature 486(7402) 774 \\ Miyake F et al. 2013 Nat. Commun. 41748 \\ Owens MJ et al. 2011 Geophys. Res. Lett. 38(19) 1 \\ Owens MJ et al. 2016a J. Geophys. Res. Sp. Phys. \\ 121(7) 6048 \\ Owens M J et al. 2016b J. Geophys. Res. Sp. Phys. \\ 121(7) 6064 \\ Owens M J et al. 2017 Sci. Rep. 741548 \\ Ramanjooloo Y 2014 Astron. \& Geophys. 551.32 \\ Ramanjooloo Y 2015 Comets as Natural Labo- \\ ratories: Interpretations of the Structure of the Inner \\ Heliosphere PhD thesis (MSSL, University College
}

London)

Roy l et al. 2016 J. Atmos. Solar-Terrestrial Phys. 149167

Steinhilber F \& Beer J 2013 J. Geophys. Res. Sp. Phys. 118(5) 1861

Stephenson FR \& Willis DM 1999 Astron. \& Geophys. $\mathbf{4 0} 6.21$

Usoskin IG et al. 2013 Astron. Astrophys. 552 L3 Usoskin IG et al. 2016 Sol. Phys. 291(9-10) 2685 Wilkinson J et al. 2016 Astron. \& Geophys. 572.37 Wolff EW et al. 2012 Geophys. Res. Lett. 39(8) 1 